firmed in a chest radiograph. Pain and dyspnoea were the commonest symptoms, and the pneumothorax usually resolved without complications. The lung tended to re-expand within a few days, so that the condition was sometimes overlooked until there had already been several attacks of unexplained pain or dyspnoea.

Eleven patients underwent operation, which made it possible to examine the thoracic contents. Foci of endimetriosis were discovered on the upper surface of the right diaphragm in four (of whom two had pelvic endometriosis and one had a pulmonary lesion), while a fifth patient, who did not have a thoracotomy, was known to have had pelvic endometriosis in the past. Actual perforation of the diaphragm was observed in two of the patients with endometriosis, while in another patient in whom it was seen it was attributed to a congenital fault. Three patients were found to have emphysematous blebs or bullae without endometriosis, but in the remaining three cases no abnormality was noted at thoracotomy.

The aetiology of this syndrome is obscure, though endometriosis, which seems to be implicated in a number of cases, could explain the peak incidence in the fourth decade and the relationship to menstruation. None of the theories put forward to account for the actual entry of air into the right pleural cavity is compatible with all the available evidence. Endometrial embolism to the lung with the rupture of a subpleural focus during menstruation was suggested by the observation that, in two patients, the first pneumothorax occurred after an operation for pelvic endometriosis. However, pulmonary endometrial lesions were found in only one patient, and haemothorax-a common event in other cases of intrathoracic endometriosis ${ }^{8}$ - was never observed. It has also been postulated that air might escape from the genital tract in patients with pelvic endometriosis and enter the pleural cavity through a hole in the right diaphragm-either a congenital fault, or a perforation associated with an endometrial implant. In keeping with this theory is the greater frequency of congenital faults of the diaphragm on the right side (a fact to which the right-sided effusion in Meigs's syndrome has also been attributed), and the actual finding of a perforated right diaphragm in three patients from the present series. On the other hand air was observed under the diaphragm on only one occasion. In three patients emphysema was the only abnormality found at thoracotomy, and in one of these histological examination of an excised emphysematous segment disclosed no sign of endometriosis. The possibility therefore remains that an emphysematous bulla is more liable to rupture at the onset of the menses than during other phases of the female hormonal cycle.

Despite the frequency of pneumothorax the prognosis is uniformly good, with a tendency to full re-expansion of the lung after each attack. In three patients the incidence of pneumothorax was greatly reduced by hormonal therapy (androgens, and oestrogens or progesterone), but the condition recurred on withdrawal of the drug. A radical cure was attempted in 11 of the 13 patients, no less than seven different methods being employed. These included excision of the diaphragmatic lesion with repair of the defect (four cases), the removal of a pleural bleb, the insertion of an intercostal tube and pleurodesis by means of scarification (two cases), talc, olive oil, or pleurectomy. In all but one of these patients a cure was apparently achieved-an interesting reflection on current controversies about the best method for preventing pneumothorax. ${ }^{9}$

\section{Autoantibodies to Colon in Ulcerative Colitis}

Ulcerative colitis is commonly considered to be one of the autoimmune diseases, partly because no other acceptable cause has been found, and partly because in some patients an antibody against a constituent of the colon can be detected. This was first shown by O. Broberger and P. Perlmann ${ }^{1}$ in the serum of juvenile patients, in whom it is far commoner than in adults. Theirs was an agglutination method, using red cells coated with an extract of foetal colon. A similar autoantibody detectable by fluorescent staining can also be induced in experimental animals by immunization with extracts of colon of another species incorporated in Freund's complete adjuvant. ${ }^{2}$ But of greater interest was the discovery by G. L. Asherson and E. J. Holborow ${ }^{3}$ that a similar antibody could be induced by the immunization of rabbits with certain strains of Escherichia coli. That the anticolon antibodies found in ulcerative colitis are the result of such bacterial immunization is further supported by the observation of Perlmann and his colleagues ${ }^{4}$ that these antibodies could be specifically inhibited by polysaccharide extracts of $E$. coli.

The pathogenic significance of these anticolon antibodies is difficult to assess. It is extremely doubtful whether they play any role in damaging the colonic mucosa, since there is no correlation between their presence and clinical severity of the disease, nor are they cytotoxic to colonic cells in tissue culture. Moreover, in rabbits with anticolon antibodies induced by immunization with appropriate strains of $E$. coli no morphological evidence of injury to the colon was obtained. A recent study, ${ }^{6}$ however, while agreeing with the earlier results, found evidence of enzymatic abnormality-notably increased alkaline phosphatase and glucose 6-phosphate dehydrogenase in the mucosal cells of the immunized animals.

Though the available evidence is against any pathogenic significance of the humoral anticolon antibodies found in ulcerative colitis, there is some evidence that specific immunological injury might be mediated by sensitized leucocytes. Thus in tissue cultures of colonic epithelium the lymphocytes from patients with colitis were consistently more cytotoxic than cells from patients with other diseases; and this effect was tissue specific. ${ }^{7}$

The evidence, therefore, that ulcerative colitis is an autoimmune disease is suggestive but not yet conclusive, especially as it is conceivable that the anticolon antibodies may be merely the result of exposure of the ulcerated areas to microorganisms fortuitously endowed with a cross-reacting antigen. But the rarity of similar antibodies in other diseases of the colon $^{5}$ does not support this interpretation.

1 Broberger, O., and Perlmann, P., 1959, f. exp. Med., 110, 657.

2 Holborow, E. J., Asherson, G. L., and Wigley, R. D., 1963, Immunology, 6, 551 .

s Asherson, G. L., and Holborow, E. J., 1966, Immunology, 10, 161.

4 Perlmann, P., Hammarström, S., Lagercrantz, R., and Gustafsson, B. E., 1965, Ann. N.Y. Acad. Sci., 124, 377.

5 Harrison, W. J., 1965, Lancet, 1, 1346.

- Cooke, E. M., Filipe, M. I., and Dawson, I. M. P., 1968, F. Path. Bact., 96, 125.

7 Perlmann, P., and Broberger, O., 1963, f. exp. Med., 117, 717.

It appears likely that owing to Post Office delays this issue of the B.M.F. will be delivered late. 